\title{
Potential health risks surrounding ingredients of pre-workout and post-workout dietary supplements: a thorough label analysis
}

\section{Potenciais riscos de saúde relacionados \\ com ingredientes de suplementos \\ alimentares de pré-treino e pós-treino: \\ análise meticulosa de rótulos}

João Nuno Alves do Vale MARQUES ${ }^{1}$ (iD) 0000-0001-9340-1783

João Paulo CAPELA ${ }^{1,2}$ (ID) 0000-0003-2607-1264

\section{A B S T R A C T}

\section{Objective}

Dietary supplements use is increasing. Dietary supplements may contain high doses of substances or dangerous ingredient combinations. This article aims to investigate, by analyzing dietary supplements labels, if there are any doping substances or dangerous amounts of any other component in the reviewed dietary supplements.

\section{Methods}

Several brands which possessed their supplements sorted in pre-workout and post-workout were analyzed. 40 dietary supplements with all ingredients described were included. The minimum and maximum dosages of dietary supplements were statistically described as Mean \pm SD.

\section{Results}

Citrus aurantium extract, Yohimbe extract, Garcinia cambogia extract and Maca root extract were reported in some of the analyzed dietary supplements. Regarding caffeine, the pre-workout group displayed higher mean caffeine

${ }^{1}$ FP-I3ID, Faculdade de Ciências da Saúde, Universidade Fernando Pessoa. Porto, Porto, Portugal. Correspondence to: JP CAPELA. E-mail: <joaoc@ufp.edu.pt>.

2 UCIBIO, REQUIMTE, Laboratório de Toxicologia, Departamento de Ciências Biológicas, Faculdade de Farmácia, Universidade do Porto, Rua de Jorge Viterbo Ferreira n. 228, 4050-313 Porto, Portugal.

This article was elaborated from the dissertation by João MARQUES, entitled "Health and career risks surrounding consumption of dietary supplements". Universidade Fernando Pessoa, 2020.

How to cite this article

Marques JNAV, Capela JP. Potential health risks surrounding ingredients of pre-workout and post-workout dietary supplements: a thorough label analysis. Rev Nutr. 2022;35:e200148. https://doi.org/10.1590/1678-9865202235e200148 
$(241 \pm 86 \mathrm{mg})$ than the post-workout group $(183 \pm 68 \mathrm{mg})$, and the minimal mean dose was $226 \pm 84 \mathrm{mg}$; meanwhile, the maximal mean dose was $242 \pm 88 \mathrm{mg}$. Concerning creatine, the pre-workout group displayed lower mean creatine $(3106 \pm 1079 \mathrm{mg})$ than the post-workout group $(4137 \pm 4177 \mathrm{mg})$, and the minimal mean dose was $3167 \pm 1728 \mathrm{mg}$; meanwhile, the maximal mean dose was $3917 \pm 3643 \mathrm{mg}$. The salt content in the post-workout group displayed a much higher mean $(2155 \pm 4486 \mathrm{mg})$ than the pre-workout group $(464 \pm 605 \mathrm{mg})$, and the minimal mean dose was $1635 \pm 3930$ mg; meanwhile, the maximal mean dose was $1708 \pm 3926 \mathrm{~g}$.

\section{Conclusion}

No doping substances were reported in the dietary supplements, but consumption recommendations on the label could lead to excessive consumption of some not yet fully tested ingredients.

Keywords: Dietary Supplements. Doping. Nutrition Sciences. Toxicity.

\section{R E S U M O}

\section{Objetivo}

O uso de suplementos alimentares está a aumentar. Estes podem conter altas doses de substâncias ou combinações de ingredientes perigosas. Este artigo procura encontrar, analisando os rótulos dos produtos, se existem substâncias dopantes ou nocivas.

\section{Métodos}

Foram analisadas várias marcas cujos respectivos suplementos foram classificados em pré e pós-treino. Foram incluídos 40 suplementos com todos os ingredientes descritos. A respectiva dose mínima e máxima foi descrita estatisticamente como média $\pm D P$.

\section{Resultados}

Extratos de Citrus aurantium, Yohimbe, Garcinia cambogia e raiz de Maca foram encontrados nos suplementos analisados. O grupo pré-treino apresentou maior média de cafeína $(241 \pm 86 \mathrm{mg})$ do que o grupo pós-treino $(183 \pm 68 \mathrm{mg})$, e a dose média mínima foi de $226 \pm 84 \mathrm{mg}$, enquanto a dose média máxima foi de $242 \pm 88 \mathrm{mg}$. O grupo pré-treino apresentou menor média de creatina $(3106 \pm 1079 \mathrm{mg})$ do que o grupo pós-treino $(4137 \pm 4177 \mathrm{mg})$, e a dose média mínima foi de

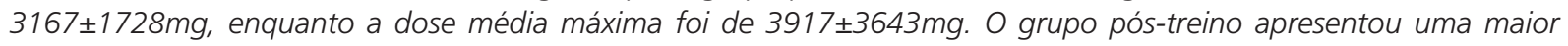
média de sal $(2155 \pm 4486 \mathrm{mg})$ do que o grupo pré-treino $(464 \pm 605 \mathrm{mg})$, e a dose média mínima foi $1635 \pm 3930 \mathrm{mg}$, enquanto a dose média máxima foi de $1708 \pm 3926 \mathrm{mg}$.

\section{Conclusão}

Não foram encontradas substâncias dopantes nos suplementos, mas algumas recomendações de consumo nos rótulos poderão levar à sobredose de certos ingredientes menos testados.

Palavras-chave: Suplementos Nutricionais. Doping. Ciências da Nutrição. Toxicidade.

\section{INTROD UCTION}

From times immemorial, the prospect of increased physical performance attained with relative lower effort always seemed appeasable. An individual who wants to improve his performance or just improve his body esthetics almost always engages in better nutrition. Dietary supplements (DS) were envisioned for athletes with increased nutritional needs, which a balanced diet could not accomplish alone, but an ever-growing market for these products lures fitness pursuers to these relatively unneeded products and their dangers [1].

The European Food Safety Authority (EFSA) represents the chief regulatory authority on dietary supplements in Europe, which regulates mineral and vitamin supplements to ensure the consumers' protection, but since dietary supplements are considered as a subcategory of food, evidence of their efficacy and safety is not the producer's obligation [1]. Subsequently, cross-contamination of doping substances 
and undeclared doping substances on product labels has become a real health problem for athletes and non-athletes. According to the World Anti-Doping Agency (WADA), which promotes the detection and prevention of doping in athletes, anti-doping rule violations have gone down approximately $16 \%$ between 2013 and $2017[2,3]$. These numbers are somewhat encouraging and seem to shadow the growing reality of DS with very high contamination rates, which comprehend a total of about $6.4 \%-8.8 \%$ of all anti-doping rule violations [4-7]. It is also known that DS are sold indiscriminately, so adolescents are being exposed on a daily basis to substances that put great strain on several organs $[4,8]$.

Usually, athletes are aware that consuming performance-enhancing drugs is cheating, and they often show anti-doping attitudes [9]. Still, a large proportion of athletes endanger their careers by not taking counsel on DS consumption from health professionals as much as they should. Instead, they consult teammates or their coach $[1,4,9]$.

This article aims to investigate if there are any doping substances or dangerous amounts of any other component stated on the labels of the analyzed DS, while also making a statement regarding possible career and health implications towards the consumer.

\section{METHODS}

Several renowned DS brands were screened: Optimum Nutrition, BSN, Biotech USA, Scitech Nutrition, Gold Nutrition, Cellucor, Nutramino, Applied Nutrition, Muscletech, Prozis. BSN and Nutramino only had pre-workout DS. Following this initial search of supplements in these brands, 50 randomly chosen Dietary Supplements (DS), currently available for online purchase on their brand's websites, were analyzed. The inclusion criteria were the following: (1) being related to some sort of physical exercise and meant to be taken accordingly; (2) having each DS had its ingredients labelled on the official brand website. We excluded all DS that did not describe appropriately their formulation, either the full formula or the amount of some substance. In fact, 10 different DS did not meet these criteria and were excluded. After refining both dietary brands and supplements, 40 dietary supplements with the full formula available on each respective brand's websites, were selected and divided into pre-workout and post-workout. This division was solely made according to the website's self-categorizing parameters, meaning that each website, according to its makers' own judgment, sorted which DS were pre- or post-workout. Pre-workout supplements are DS that are meant to be taken before the workout. Although there are no fixed constituents for pre-workout DS, most include stimulants aiming for higher performance. Post-workout supplements are DS that supposedly do most benefit when consumed after the workout, aiming for a swift recovery. Most of these have amino acids, vitamins, and small amounts of carbohydrates.

Since the recommended dose differed in some of the DS, and some DS had two recommended doses, initially, only the minimal recommended dose was considered for all the DS, and later on, the maximal dosage was analyzed. For each DS the following categories were made to compare each other: recommended dose, energy, caffeine, creatine, amino acids and derivates, macronutrients and derivates (lipids, protein, carbohydrates, and fiber), vitamins, minerals and derivates, vegetable extracts, and other ingredients. Every category was analyzed in milligrams except for the recommended dose and macronutrients and derivates, which were described in grams. DS were screened for banned substances according to WADA's list of prohibited substances, and any dangerous amounts of those substances were noted.

Given that some DS displayed two alternative doses, i.e., a minimal dose and a maximal dose, we calculated and statistically analyzed the minimum and maximum dosage of all reviewed DS' recommended 
dose, and also for some important ingredients such as caffeine, creatine and salt content. Data were statistically analyzed using GraphPad Prism 8 software (GraphPad Software, CA, USA). Data passed at least one normality test (either the Shapiro-Wilk test or the Kolmogorov-Smirnov test) and Mean and SD were calculated.

\section{RE S U L T S}

A total of 40 DS were deemed appropriate for further analysis, given that the full formula was completely described. The DS included in this article belonged to 10 different brands with the number of products within brackets: Optimum Nutrition, USA [3], BSN, USA [1], Biotech USA, USA [4], Scitec Nutrition, USA [7], Gold Nutrition, Portugal [6], Cellucor, USA [3], Nutramino, Denmark [1], Applied Nutrition, UK [4], Muscletech, USA [8], Prozis, Portugal [3]. The DS were analyzed by dividing them into two groups in accordance with the manufacturer's own judgment: pre- and post-workout.

In Table 1, the description of minerals and vegetable extracts constituents is exhibited. The full list of all ingredients can be seen in Supplementary table 1: which is deposited at Zenodo OpenAIRE project (https://doi.org/10.5281/zenodo.5060047). Relating to the amino acids content, the post-workout group displayed a higher mean number of amino acids and derivates, 6 , than the pre-workout group, 4 . Concerning the vitamin content, the post-workout group displayed a higher mean number of vitamins and derivates, 4, than the pre-workout group, 2. Regarding vegetable extracts in each group, the pre-workout group displayed a higher mean number of these extracts, 3, than the post-workout group, 1. Lastly, both pre and post-workout groups had the same mean number of uncategorized ingredients, 2.

Table 1 - Description of the most relevant ingredients contained in the dietary supplements, sorted in several columns describing their recommended dose, energy, minerals and derivates and vegetable extracts.

\begin{tabular}{|c|c|c|c|c|}
\hline Product & Dose & Energy(kcal) & Minerals and derivates & Vegetable Extracts \\
\hline \multicolumn{5}{|c|}{ Pre-Workout } \\
\hline 1 & $10 \mathrm{~g}$ & 10 & & $\begin{array}{l}\text { 25mg (AstraGin -Astragalus membranaceus Extract and } \\
\text { Panax notoginseng Extract) }\end{array}$ \\
\hline 2 & $13 \mathrm{~g}$ & 5 & $\begin{array}{l}0.170 \mathrm{mg} \text { Sodium }+160 \mathrm{mg} \text { Potassium + } \\
70 \mathrm{mg} \text { Calcium }\end{array}$ & 150mg Longan Extract(fruit) + 26mg Yohimbe Extract(bark) \\
\hline 3 & $9.5 \mathrm{~g}$ & 3 & 20mg Salt & \\
\hline 4 & $4 \mathrm{~g}$ & 2,4 & & $12 \mathrm{mg}$ Green tea leaves extract $+11 \mathrm{mg}$ chlorogenic acid \\
\hline 5 & $9.5 \mathrm{~g}$ & & & $\begin{array}{l}250 \mathrm{mg} \text { Green tea extract }+250 \mathrm{mg} \text { Beets extract }+86 \mathrm{~g} \\
\text { Cocoa seed extract }+50 \mathrm{mg} \text { AstraGin }\end{array}$ \\
\hline 6 & $30 \mathrm{~g}$ & & $\begin{array}{l}0.006 \mathrm{mg} \text { Chromium }+56 \mathrm{mg} \text { Magnesium }+ \\
10 \mathrm{mg} \text { Calcium(AKG)+ } 0.053 \mathrm{mg} \text { Chromium } \\
\text { Piculinate }\end{array}$ & $\begin{array}{l}\text { 10mg Garcinia cambogia extract(Sibutramine) }+400 \mathrm{mg} \\
\text { Citrus aurantium extract (synephrine) }+10 \mathrm{mg} \text { Bacopa } \\
\text { monnieri extract }+10 \mathrm{mg} \text { ginger extract }+10 \mathrm{mg} \text { guarana } \\
\text { extract }+100 \mathrm{mg} \text { grape seed extract }+10 \mathrm{mg} \text { sour melon } \\
\text { extract }+10 \mathrm{mg} \text { BioPerine }{ }^{\circledast} \text { Piper }\end{array}$ \\
\hline 7 & $20 \mathrm{~g}$ & & 56mg Magnesium & $\begin{array}{l}200 \mathrm{mg} \text { Green tea extract }+20 \mathrm{mg} \text { grape seed extract }+ \\
5 \mathrm{mg} \text { Bioperine (Piperine) }\end{array}$ \\
\hline 8 & $7 \mathrm{~g}$ & & & 50mg Rhodiola Rosea extract \\
\hline 9 & $50 \mathrm{~g}$ & 154 & $\begin{array}{l}\text { 540mg Sodium }+1400 \mathrm{mg} \text { Salt }+0.012 \mathrm{mg} \\
\text { Chromium }++0.1 \mathrm{mg} \text { Chromium Picolinate }\end{array}$ & $\begin{array}{l}100 \mathrm{mg} \text { Ginseng extract }+50 \mathrm{mg} \text { grape seed extract }+50 \mathrm{mg} \text { ginger } \\
\text { extract }\end{array}$ \\
\hline 10 & $22 \mathrm{~g}$ & & & 200mg Maca root extract + 125mg Guarana extraxt \\
\hline 11 & $16 \mathrm{~g}$ & 5 & & $\begin{array}{l}62.5 \mathrm{mg} \text { Theacrine }+1 \mathrm{mg} \text { Rauwolfia vomitoria extract }+ \\
0.05 \mathrm{mg} \text { Huperzine A }\end{array}$ \\
\hline
\end{tabular}


Table 1 - Description of the most relevant ingredients contained in the dietary supplements, sorted in several columns describing their recommended dose, energy, minerals and derivates and vegetable extracts.

2 of 3

\begin{tabular}{|c|c|c|c|c|}
\hline Product & Dose & Energy(kcal) & Minerals and derivates & Vegetable Extracts \\
\hline 12 & $10.5 \mathrm{~g}$ & 5 & & 150mg Grape seed extract \\
\hline 13 & $60 \mathrm{~g}$ & 17 & & \\
\hline 14 & $10.5 \mathrm{~g}$ & 1 & $0.319 m g$ Salt & 100mg Theacrine \\
\hline 15 & $15 \mathrm{~g}$ & 3 & $171 \mathrm{mg}$ Sodium + 426mg Salt & 50mg Astragin + 5mg Bioperine \\
\hline 16 & $21 \mathrm{~g}$ & 35 & & $\begin{array}{l}2500 \mathrm{mg} \text { Betaine anhydrous }+200 \mathrm{mg} \text { Hawthorn extract } \\
+100 \mathrm{mg} \text { Hovenia dulcis extract }+30 \mathrm{mg} \text { Yohimbe extract }\end{array}$ \\
\hline 17 & $6 g$ & & 15mg Calcium & 50mg Rhodiola extract + 20mg Yohimbe extract \\
\hline 18 & $\log$ & 20 & & $\begin{array}{l}500 \mathrm{mg} \text { ViNitrox (grape extract and apple extract) }+400 \mathrm{mg} \\
\text { C. canephora robusta extract }+250 \mathrm{mg} \text { grape extract } \\
+100 \mathrm{mg} \text { Black currant extract }+100 \mathrm{mg} \text { Goji extract }+ \\
40 \mathrm{mg} \text { Theacrine }+40 \mathrm{mg} \text { Grains of paradise extract }\end{array}$ \\
\hline 19 & $18 \mathrm{~g}$ & & 390mg Sodium + 60mg Calcium & $\begin{array}{l}250 \mathrm{mg} \text { Grape extract }+100 \mathrm{mg} \text { Nutmeg extract }+100 \mathrm{mg} \\
\text { Jujube extract }+75 \mathrm{mg} \text { Skullcap extract }+75 \mathrm{mg} \text { Theacrine }\end{array}$ \\
\hline 20 & $15 g$ & & & $\begin{array}{l}\text { 150mg Paullinia Cupana seed extracts }+75 \mathrm{mg} \text { Panax } \\
\text { ginseng root extract }+7.5 \mathrm{mg} \text { Salvia miltiorrhiza }+3.8 \mathrm{mg} \\
\text { Ophiopogon japonicus }+15 \mathrm{mg} \text { Pinus massoniana + } \\
4.50 \mathrm{mg} \text { Ginko biloba }\end{array}$ \\
\hline 21 & $6.5 \mathrm{~g}$ & & & \\
\hline \multicolumn{5}{|c|}{ Post-Workout } \\
\hline 22 & $\log$ & 15 & $\begin{array}{l}\text { 100mg Sodium + 100mg Potassium + 60mg } \\
\text { Magnesium }\end{array}$ & $250 \mathrm{mg}$ Bakers yeast beta glucan $+200 \mathrm{mg}$ Rhodiola extract \\
\hline 23 & $334 \mathrm{~g}$ & 1250 & $\begin{array}{l}430 \mathrm{mg} \text { Sodium }+940 \mathrm{mg} \text { Potassium + } \\
5 \mathrm{mg} \text { Iron }+0.12 \mathrm{mg} \text { Chromium }+140 \mathrm{mg} \\
\text { Magnesium }+0.075 \mathrm{mg} \text { Molybdenum + } \\
2 \mathrm{mg} \text { Manganese }+1 \mathrm{mg} \text { Copper }+0.07 \mathrm{mg} \\
\text { Selenium + } 15 \mathrm{mg} \text { Zinc }+0.15 \mathrm{mg} \text { lodine + } \\
\text { 460mg Phosphorus }+630 \mathrm{mg} \text { Calcium }\end{array}$ & \\
\hline 24 & $9.4 \mathrm{~g}$ & 3 & $4 \mathrm{mg}$ Salt $+5.9 \mathrm{mg}$ Zinc & \\
\hline 25 & $11.5 \mathrm{~g}$ & 7 & Under $1 \mathrm{mg}$ Salt & 164mg Betaine hydrochoride \\
\hline 26 & $162 \mathrm{~g}$ & 588 & $\begin{array}{l}\text { 380mg Salt }+360 m g \text { Potassium }+58.9 m g \\
\text { Magnesium + 20mg Calcium(AKG) }\end{array}$ & $5 \mathrm{mg}$ Bioperine \\
\hline 27 & $140 \mathrm{~g}$ & 447 & $\begin{array}{l}\text { 1000mg Salt }+445 \mathrm{mg} \text { Potassium }+0.012 \mathrm{mg} \\
\text { Chromium }+57.3 \mathrm{mg} \text { Magnesium }+1.5 \mathrm{mg} \\
\text { Zinc }+10 \mathrm{mg} \text { Calcium(AKG) }\end{array}$ & $\begin{array}{l}500 \mathrm{mg} \text { Avena sativa extract }+100 \mathrm{mg} \text { Coleus forskohlii } \\
\text { extract }+100 \mathrm{mg} \text { Sour melon extract }+25 \mathrm{mg} \text { Bromelain }+ \\
25 \mathrm{mg} \text { Papain }+5 \mathrm{mg} \text { Bioperine }\end{array}$ \\
\hline 28 & $50 \mathrm{~g}$ & 186 & 140mg Salt & \\
\hline 29 & $78 g$ & 282 & $\begin{array}{l}\text { 195mg Sodium }+15000 \mathrm{mg} \text { Salt }+0.162 \mathrm{mg} \\
\text { Chromium }+192 \mathrm{mg} \text { Magnesium }+1.5 \mathrm{mg} \\
\text { Zinc }+225 \mathrm{mg} \text { Calcium }+155 \mathrm{mg} \text { Chloride }\end{array}$ & $37.5 \mathrm{mg}$ Grape seed extract + 23mg Ginkgo biloba \\
\hline 30 & $52 \mathrm{~g}$ & 205 & $\begin{array}{l}\text { 198mg Sodium }+490 \mathrm{mg} \text { Salt }+162 \mathrm{mg} \\
\text { Phosphorus }+247 \mathrm{mg} \text { Calcium }\end{array}$ & 173mg Bromelain + 173mg Papain \\
\hline 31 & $320 \mathrm{~g}$ & 1225 & $\begin{array}{l}\text { 1179mg Sodium }+2950 m g \text { Salt }+2925 m g \\
\text { Potassium }+118 m g \text { Magnesium }+1.8 m g \\
\text { Zinc }+978 m g \text { Phosphorus }+623 m g \text { Calcium }\end{array}$ & \\
\hline 32 & $75 g$ & 305 & $\begin{array}{l}80 \mathrm{mg} \text { Salt }+0.016 \mathrm{mg} \text { Chromium }+7.9 \mathrm{mg} \\
\text { Manganese }+2.4 \mathrm{mg} \text { Zinc }\end{array}$ & \\
\hline 33 & $11.7 \mathrm{~g}$ & & 360mg Calcium & \\
\hline 34 & $6 \mathrm{~g}$ & & & \\
\hline 35 & $100 \mathrm{~g}$ & 353 & $\begin{array}{l}400 m g \text { Sodium }+1000 m g \text { Salt }+600 m g \\
\text { Potassium } 113 m g+\text { Magnesium }+240 m g \\
\text { Calcium }\end{array}$ & \\
\hline 36 & $10 \mathrm{~g}$ & 30 & 100mg Sodium + 18mg Potassium & $25 \mathrm{mg}$ Green tea extract \\
\hline 37 & $48 g$ & 150 & $\begin{array}{l}35 \mathrm{mg} \text { Sodium }+24 \mathrm{mg} \text { Potassium }+32.5 \mathrm{mg} \\
\text { Magnesium }\end{array}$ & \\
\hline
\end{tabular}


Table 1 - Description of the most relevant ingredients contained in the dietary supplements, sorted in several columns describing their recommended dose, energy, minerals and derivates and vegetable extracts.

3 of 3

\begin{tabular}{|c|c|c|c|c|}
\hline Product & Dose & Energy(kcal) & Minerals and derivates & Vegetable Extracts \\
\hline 38 & $1.7 \mathrm{~g}$ & & & \\
\hline 39 & $11 \mathrm{~g}$ & & 120mg Sodium + 0.1mg Chromium & $\begin{array}{l}2500 \mathrm{mg} \text { Betaine anhydrous }+800 \mathrm{mg} \text { CinDura } \\
\text { (Cinnamomum tamala leaf extract and Garcinia } \\
\text { mangostana fruit rind extract) }+5.3 \mathrm{mg} \text { Bioperine }\end{array}$ \\
\hline 40 & $80 \mathrm{~g}$ & 299 & $\begin{array}{l}780 \mathrm{mg} \text { Salt }+480.8 \mathrm{mg} \text { Potassium }+232 \mathrm{mg} \\
\text { Magnesium }+310.4 \mathrm{mg} \text { Sodium }\end{array}$ & $\begin{array}{l}\text { 500mg Prunus Cerasus + 500mg Lythraceae punica granatum } \\
+250 \mathrm{mg} \text { Curcumin complex }\end{array}$ \\
\hline
\end{tabular}

In tables 2 and 3 , all of the most relevant constituents were thoroughly screened. Each dose hence stated is a daily dose. Only 5 DS from the entire samples displayed an alternative dose, to which a maximal and minimal dose was obtained. Regarding caffeine, the pre-workout group displayed a higher mean caffeine dose $(241 \pm 86 \mathrm{mg})$ than the post-workout group $(183 \pm 68 \mathrm{mg})$, being present in 19 of the former DS group in comparison to 3 of the latter. The minimal mean dose was $226 \pm 84 \mathrm{mg}$, and the maximal mean dose was $242 \pm 88 \mathrm{mg}$, being the maximal dose of $380 \mathrm{mg}$ found in supplement number 17 . Caffeine doses in some DS could be higher than these figures since green tea extract and other vegetable extracts were often depicted in the ingredient lists, withheld varying amounts of caffeine, therefore, were not accounted for in the previous analysis. Concerning creatine, the pre-workout group displayed lower mean creatine $(3106 \pm 1079 \mathrm{mg})$ than the post-workout group $(4137 \pm 4177 \mathrm{mg})$. The minimal mean dose was $3167 \pm 1728 \mathrm{mg}$, and the maximal mean dose was $3917 \pm 3643 \mathrm{mg}$, being the maximal dose of 20000 mg found in supplement number 37. As for the salt content, antithetically to what was seen in the caffeine dosage distribution within groups, the post-workout group displayed a much higher mean salt dosage $(2155 \pm 4486 \mathrm{mg})$ than the pre-workout group $(464 \pm 605 \mathrm{mg})$. The minimal mean dose was $1635 \pm 3930$ $\mathrm{mg}$, and the maximal mean dose was $1708 \pm 3926 \mathrm{mg}$, being the maximal dose of $15000 \mathrm{mg}$ found in supplement number 29.

Table 2 - Minimum and maximum dosage options portrayed by the Dietary supplements brands on the most relevant ingredients (Caffeine, Creatine, Yohimbe extract, Citrus aurantium, Salt).

1 of 2

\begin{tabular}{|c|c|c|c|c|c|c|c|c|}
\hline \multirow{2}{*}{ Product } & \multicolumn{2}{|c|}{ Recommendeddose (g) } & \multicolumn{2}{|c|}{ Caffeine (mg) } & \multicolumn{2}{|c|}{ Creatine (mg) } & \multicolumn{2}{|c|}{ Salt (mg) } \\
\hline & Minimum & Maximum & Minimum & Maximum & Minimum & Maximum & Minimum & Maximum \\
\hline \multicolumn{9}{|c|}{ Pre-Workout Supplements } \\
\hline 1 & 10 & 10 & 175 & 175 & 3000 & 3000 & & \\
\hline 2 & 13 & 13 & 300 & 300 & 4000 & 4000 & & \\
\hline 3 & 9.5 & 19 & 150 & 300 & 1500 & 3000 & 20 & 40 \\
\hline 4 & 4 & 4 & 43 & 43 & & & & \\
\hline 5 & 9.5 & 9.5 & 300 & 300 & & & & \\
\hline 6 & 30 & 30 & 350 & 350 & 5473 & 5473 & & \\
\hline 7 & 20 & 20 & 300 & 300 & 3929 & 3929 & & \\
\hline 8 & 7 & 7 & 200 & 200 & 2641 & 2641 & & \\
\hline 9 & 50 & 50 & 150 & 150 & 4000 & 4000 & 1400 & 1400 \\
\hline 10 & 22 & 22 & 250 & 250 & 3800 & 3800 & & \\
\hline 11 & 16 & 16 & 300 & 300 & 2000 & 2000 & & \\
\hline 12 & 10.5 & 10.5 & & & & & & \\
\hline 13 & $60 \mathrm{ml}$ & $60 \mathrm{ml}$ & 200 & 200 & & & & \\
\hline 14 & 10.5 & 10.5 & 200 & 200 & 3250 & 3250 & 0.319 & 0.319 \\
\hline
\end{tabular}


Table 2 - Minimum and maximum dosage options portrayed by the Dietary supplements brands on the most relevant ingredients (Caffeine, Creatine, Yohimbe extract, Citrus aurantium, Salt).

2 of 2

\begin{tabular}{|c|c|c|c|c|c|c|c|c|}
\hline \multirow{2}{*}{ Product } & \multicolumn{2}{|c|}{ Recommendeddose (g) } & \multicolumn{2}{|c|}{ Caffeine (mg) } & \multicolumn{2}{|c|}{ Creatine (mg) } & \multicolumn{2}{|c|}{ Salt (mg) } \\
\hline & Minimum & Maximum & Minimum & Maximum & Minimum & Maximum & Minimum & Maximum \\
\hline \multicolumn{9}{|c|}{ Pre-Workout Supplements } \\
\hline 15 & 15 & 15 & 250 & 250 & 3000 & 3000 & 426 & 426 \\
\hline 16 & 21 & 21 & 350 & 350 & 3000 & 3000 & & \\
\hline 17 & 6 & 12 & 160 & 380 & 1500 & 3000 & & \\
\hline 18 & 10 & 10 & 350 & 350 & & & & \\
\hline 19 & 18 & 18 & 300 & 300 & & & & \\
\hline 20 & 15 & 15 & 150 & 150 & 3000 & 3000 & & \\
\hline 21 & 6.5 & 6.5 & 160 & 160 & 1000 & 1000 & & \\
\hline \multicolumn{9}{|c|}{ Post-Workout Supplements } \\
\hline 22 & 10 & 10 & & & & & & \\
\hline 23 & 334 & 334 & & & 1000 & 1000 & & \\
\hline 25 & 11.5 & 11.5 & 250 & 250 & 1760 & 1760 & 1 & 1 \\
\hline 26 & 162 & 162 & & & 5763 & 5763 & 380 & 380 \\
\hline 27 & 140 & 140 & & & 4858 & 4858 & 1000 & 1000 \\
\hline 28 & 50 & 50 & & & 1056 & 1056 & 140 & 140 \\
\hline 29 & 78 & 78 & & & & & 15000 & 15000 \\
\hline 30 & 52 & 52 & & & & & 490 & 490 \\
\hline 31 & 320 & 320 & & & & & 2950 & 2950 \\
\hline 32 & 75 & 75 & & & 2300 & 2300 & 80 & 80 \\
\hline 33 & 11.7 & 11.7 & & & 7000 & 7000 & & \\
\hline 34 & 6 & 7 & & & & & & \\
\hline 35 & 100 & 200 & & & & & 1000 & 2000 \\
\hline 36 & 10 & 10 & 100 & 100 & & & & \\
\hline 37 & 48 & 192 & & & 5000 & 20000 & & \\
\hline 40 & 80 & 80 & & & & & & \\
\hline
\end{tabular}

Table 3 - Mean and standard deviation of all reviewed Dietary supplements' recommended dose, caffeine, creatine and salt.

\begin{tabular}{|c|c|c|c|c|c|c|c|c|}
\hline \multirow{2}{*}{ Variables } & \multicolumn{2}{|c|}{ Recommended dose } & \multicolumn{2}{|c|}{ Caffeine (mg) } & \multicolumn{2}{|c|}{ Creatine (mg) } & \multicolumn{2}{|c|}{ Salt (mg) } \\
\hline & Min & Max & Min & Max & Min & Max & Min & Max \\
\hline Pre-workout DS by & $15 \pm 10$ & $16 \pm 10$ & $232 \pm 85$ & $250 \pm 88$ & $3006 \pm 1174$ & $3206 \pm 1006$ & $462 \pm 656$ & $467 \pm 651$ \\
\hline minimum and maximum & $(10 ; 20)$ & $(11 ; 21)$ & $(192 ; 272)$ & $(109 ; 291)$ & $(2356 ; 3657)$ & $(2649 ; 3763)$ & $(-582 ; 1505)$ & $(-570 ; 1503)$ \\
\hline Pre-workout total & \multicolumn{2}{|c|}{$\begin{array}{l}16 \pm 10 \\
(12 ; 19)\end{array}$} & \multicolumn{2}{|c|}{$\begin{array}{c}241 \pm 86 \\
(214 ; 269)\end{array}$} & \multicolumn{2}{|c|}{$\begin{array}{l}3106 \pm 1079 \\
(2703 ; 3509)\end{array}$} & \multicolumn{2}{|c|}{$\begin{array}{l}464 \pm 605 \\
(-42 ; 970)\end{array}$} \\
\hline $\begin{array}{l}\text { Post-workout DS by } \\
\text { minimum and maximum }\end{array}$ & $\begin{array}{c}79 \pm 99 \\
(32 ; 127)\end{array}$ & $\begin{array}{l}92 \pm 105 \\
(42 ; 143)\end{array}$ & $\begin{array}{l}183 \pm 86 \\
(-6 ; 373)\end{array}$ & $\begin{array}{l}183 \pm 76 \\
(-6 ; 373)\end{array}$ & $\begin{array}{l}3387 \pm 2333 \\
(1820 ; 4954)\end{array}$ & $\begin{array}{c}4887 \pm 5474 \\
(1210 ; 8564)\end{array}$ & $\begin{array}{c}2105 \pm 4617 \\
(-1198 ; 5407)\end{array}$ & $\begin{array}{l}2205 \pm 4601 \\
(-1087 ; 5496)\end{array}$ \\
\hline Post-workout total & \multicolumn{2}{|c|}{$86 \pm 101$} & \multicolumn{2}{|c|}{$183 \pm 68$} & \multicolumn{2}{|c|}{$4137 \pm 4177$} & \multicolumn{2}{|c|}{$2155 \pm 4486$} \\
\hline
\end{tabular}

Note: DS: Dietary Supplements.

Other ingredients were not statistically analyzed in Table 2, because only a few DS contained these ingredients. Citrus aurantium extract was found in 1 pre-workout DS with a dose of $400 \mathrm{mg}$. As for yohimbe extract, it was found in 3 pre-workout supplements, and the minimal mean dose was $25 \pm 5 \mathrm{mg}$, and the maximal mean dose was $32 \pm 7 \mathrm{mg}$ (maximal dose was $40 \mathrm{mg}$ ). Garcinia cambogia extract was found in one pre-workout DS with a dose of $10 \mathrm{mg}$. Maca root extract was found in one pre-workout DS with a dose of $200 \mathrm{mg}$. 


\section{DISCUSSION}

In the pre-workout group of the "Vegetable Extracts" category, Citrus aurantium extract, Yohimbe extract, Garcinia cambogia extract, and Maca root extract were found. Maca root extract has been said by the Australian Sports Anti-Doping Authority to have a high risk of contamination and is considered by the Australian Institute of Sport a group D supplement [10]. In several of the reviewed DS, some vegetable mixtures did not state all of their components, since it was a patented formula, such as Bioperine found in $13 \%$ of all analyzed DS.

The most serious safety concern relative to contamination of DS is the potential for liver damage, and to help circumvent this issue, one must always use authenticated plants and apply good agricultural practices [11]. Also, vegetable extracts can play a role in drug efficacy by increasing serum drug concentrations to toxic levels or by reducing its action. According to preclinical interactions, Panax Ginseng, Ginkgo Biloba extract, green tea extract, grape seed, and Yohimbe, inhibit some Cytochrome P450 (CYP) enzymes [12]. All of the referred plants and extracts were found in several DS of this study.

Synephrine is the main active compound in Citrus aurantium extract. Nowadays, synephrine is often used in DS formulations related to weight loss [13]. There are ingredient associations of synephrine with caffeine made to boost the weight loss ability [14]. This is somewhat concerning since high increases in blood pressure are seen when synephrine is taken with caffeine [15]. However, synephrine only seems to produce cardiovascular effects at doses up to $100 \mathrm{mg}$, and some also state that the consumption of synephrine alone does not raise blood pressure $[16,17]$. Studies in mice also found that synephrine can cause toxin-induced liver injury in moderate amounts $(\geq 100 \mathrm{mg})$, eventually resulting in death with increased dosage amounts $(\geq 350 \mathrm{mg}$ ). The gender of the mice also played an important role in these studies, since liver toxicity and lethal amounts seemed to differ between males and females [18-20]. In this study, only one DS had synephrine in the form of Citrus aurantium extract $(400 \mathrm{mg})$, but these DS also had $350 \mathrm{mg}$ of caffeine, increasing the possibility of unwanted cardiovascular side effects. However, DS tend to have an increased amount of caffeine and a decreased amount of synephrine often misled by the amounts stated in the supplement label [21]. Perhaps this could be seen as an advantage to consumers since there is evidence that synephrine could be dangerous in certain situations. It would seem wise to restrict the use of these combined ingredients until solid clinical evidence is at hand.

Yohimbe extract or yohimbine is most often used in DS associated with an increase in physical and sexual performance. The use of this ingredient is most concerning since using DS with yohimbe bark extract according to its consumption suggestion on the label; consumers have a high probability (79\%) of consuming different amounts of yohimbe than those stated on the label. This leads to the product not wielding the wanted effects due to low dosage or having unwanted side effects related to higher than recommended dosage [22]. Only with ingestion of 15-30mg were blood pressure and anxiety increased. While taking yohimbine during exercise, mean arterial blood pressure, heart rate, norepinephrine, and effort sensation all become significantly increased. In patients taking tricyclic antidepressants, $4 \mathrm{mg}$ three times a day is enough to generate hypertension and other health issues in some individuals who are mentally ill. Overdose seems to be achieved around 200mg or higher [23]. Taking this into account, even the lowest amount of yohimbe found in DS $(20 \mathrm{mg})$ in this study poses a health threat to normotensive individuals.

Several studies confirm weight management properties in Garcinia cambogia extract when supplemented to obese or overweight individuals $[24,25]$. In several clinical trials, this plant was deemed safe to use in the treatment of obesity. Nonetheless, it has been proven that Garcinia cambogia displays inhibitory effects in CYP2B6 [26]. Despite the relative safety of this ingredient, there have been some disturbing clinical cases that raise suspicion on its use [27]. Due to these setbacks, some say it would be 
wise to avoid the ingredient completely, as it may cause harm to the user, and the results regarding weight lowering properties are scarce [25].

Garcinia cambogia extract often displays repetitive association with sibutramine, which is a known doping substance [28]. Sibutramine is a drug intended for the treatment of obesity, which was withdrawn from Europe and the United States given the potential for several cardiovascular problems [29]. Only one DS in this study had Garcinia cambogia in its ingredient list. Its daily dose was of $10 \mathrm{mg}$, which even taken several times a day is far from being considered a dangerous amount. However, if a trace of sibutramine is caught in a doping test, this could mean a several-year ban from official competitions and, in some scenarios, a denied possibility for future re-entry in the sport.

Caffeine is without a doubt a major ingredient in most DS (91\% of all reviewed pre-workout DS had caffeine). Only $16 \%$ of the post-workout DS featured caffeine, which is completely understandable since taking caffeine after exercise does not help with recovery and can lead to increased anxiety [30]. On the other hand, taking caffeine before physical trials can lead to beneficial ergogenic effects such as increased strength and sprint outcomes during a short time and lower completion time of the set course [31,32]. Although 4 to $6 \mathrm{mg} / \mathrm{kg}$ of caffeine per day seems to have no adverse diuretic consequences in healthy adults consuming caffeine regularly, this may not be the same for an athlete who consumes caffeine before the trial [33].

The acute intake of caffeine can increase blood pressure, generate heart arrhythmias and neuroendocrine side effects [33]. Due to these known side effects, the daily recommended limit of caffeine is around $400 \mathrm{mg} /$ day in adults used to caffeine consumption [33]. However, as in other articles, this is easily surpassed in many DS in this study, where one dose can reach up to $380 \mathrm{mg}[13,34]$. Additionally, there are 85 known drugs that interact with caffeine, 11 of these can result in severe interactions [33]. It seems that by following current caffeine dosage guidelines and recommendations on supplementation, there appears to be no major health consequences regarding caffeine ingestion [31].

Creatine is an amino acid compound known to improve performance when taken, from increased overall mean power and peak mean power outputs to increased time until exhaustion [35]. This might be why a great portion of athletes seem to partake in the consumption of creatine [36]. The importance and effectiveness of creatine are well represented in the analyzed DS, since this was the most used ingredient, present in $65 \%$ of all DS. Meanwhile, it was not fully understood why DS developers would pair so many of their products with caffeine and creatine (17 DS displayed both), since caffeine consumption during creatine loading can lead to decreased creatine beneficial effects, such as better muscle relaxation time [31].

A normal diet contains about 1-2g/day of creatine, which takes up to $60-80 \%$ of creatine stores. Dietary supplementation serves to complement the missing percentage [36]. According to the International Society of Sports Nutrition, creatine supplementation can be made within two phases, the maintenance phase and the loading phase, or just by a prolonged loading phase, according to the individual's goal and sport [37]. The loading phase consists of the consumption of $0.3 \mathrm{~g} / \mathrm{kg}$ per day for $5-7$ days, before maintenance at $0.03 \mathrm{~g} / \mathrm{kg}$ per day for $4-6$ weeks $[36,38]$. Creatine loading increases urinary concentrations of toxic substances, which can have a harmful effect on kidney function [38]. However, it has been found to help maintain a good hydration status due to creatine's initial fluid retention phase [36].

As far as dosage is concerned, in the reviewed DS there is a serious discrepancy between the minimum ( $3167 \mathrm{mg}$ ) and the maximum mean creatine dose (20000 mg). If the individual taking the DS with the higher dose weighs $70 \mathrm{~kg}$, then he would have the ideal daily dose for the loading phase $(0.29 \mathrm{~g} / \mathrm{kg})$. But since the loading phase is only to last about 5-7 days, then the individual would have to change to another DS with 
a lower creatine dosage. There seems to be some sort of misinformation as far as creatine dosage in some DS. Regarding creatine safety, $0.3 \mathrm{~g} / \mathrm{kg}$ to $0.8 \mathrm{~g} / \mathrm{kg}$ per day poses no health threats, and in general, it appears that creatine is a relatively safe and effective substance to integrate with DS $[31,36,38,39]$.

In any sport, sodium is important for water regulation and fluid balance, as well as good for muscle function and nerve cell activity. When sweating heavily, sodium loss can be more than $0.5 \mathrm{~g}$ and require increased intake [40].

Since it is understood that sweat loss can vary greatly between exercise types, some DS salt values might be too high for the average gym-goer who naturally already consumes too much dietary salt. In fact, the post-workout mean salt might only be adequate for aerobic athletes who train every day for several hours. One of the most alarming salt values was of $15 \mathrm{~g}$ (supplement 29), which represents 3 times the maximum amount of salt intake for normotensive individuals.

While some might say that doping substances are not necessarily harmful to their users, doping regulations are placed to prevent damage to both the individual and the competitiveness of the sport. That said, in this study the risk of ingesting sibutramine or any other prohibited substance due to contamination is high. Taking this into account, it is unadvised for athletes to take DS without proper opinion from the team doctor or nutritionist.

The amount of possible drug interactions and enzyme inhibition within the ingredients found in this study was staggering, with a high probability of consumption of DS that could lead to some CYP enzymes being inhibited. To this degree, most possible interactions with drugs were on the ingredients in the "Vegetable extracts" group, so caution in this group of ingredients would be advised when buying DS. There have also been reports of toxic events related to DS consumption leading to severe symptoms of liver toxicity $[11,41,42]$.

While this study does a great job in discriminating individual compounds on the labels of the reviewed supplements, it was agreed that its main limitation was the lack of HPLC analysis in order to find substances that are not stated on the labels.

\section{CONCLUSION}

Several DS had untested ingredients that when paired up could cause severe health issues. Although some ingredient doses were not concerning, consumption recommendations on the label could lead to dosage abuse. It is advisable to contact a nutritionist to avoid DS risks.

CONTRIBUTORS

JNA MARQUES was responsible for the data collection and manuscript writing. JP CAPELA was responsible for the study design and statistical analysis, assisted with the results organization and manuscript review.

\section{REFERE N CES}

1. Garthe I, Maughan RJ. Athletes and supplements: Prevalence and perspectives. Int J Sport Nutr Exerc Metab. 2018;28(2):126-38.

2. World Anti-doping Agency. 2013 Anti-Doping Rule Violations (ADRVs) Report. 2015, Montreal, Canada. 
3. World Anti-doping Agency. 2017 Anti-Doping Rule Violations (ADRVs) Report. 2019, Montreal, Canada.

4. Tsarouhas K, Kioukia-Fougia N, Papalexis P, Tsatsakis A, Kouretas D, Bacopoulou F, et al. Use of nutritional supplements contaminated with banned doping substances by recreational adolescent athletes in Athens, Greece. Food Chem Toxicol. 2018;115:447-50. https://doi.org/10.1016/j.fct.2018.03.043

5. Martínez-Sanz JM, Sospedra I, Ortiz CM, Baladía E, Gil-Izquierdo A, Ortiz-Moncada R. Intended or unintended doping? A review of the presence of doping substances in dietary supplements used in sports. Nutrients. 2017;9(10):1-22.

6. Helle C, Sommer A, Syversen P, Lauritzen F. Doping substances in dietary supplements. Tidsskr Den Nor Legeforening. 2019 [cited 2020 Jun 21];139(4). Available from: www.supplement411.org

7. Outram S, Stewart B. Doping through supplement use: a review of the available empirical data. Int I Sport Nutr Exerc Metab. 2015;25(1):54-9.

8. La Gerche A, Brosnan MJ. Cardiovascular effects of performance-enhancing drugs. Circulation. 2017 Jan 3 [cited 2020 Jun 21];135(1):89-99. Available from: https://pubmed.ncbi.nlm.nih.gov/28028062/

9. Mudrak J, Slepicka P, Slepickova I. Sport motivation and doping in adolescent athletes. Plos One. 2018 [cited 2020 Jun 21];13(10). Available from: https://pubmed.ncbi.nlm.nih.gov/30286200/

10. Group A. Australian Institute of Sport. [cited 2020 Mar 28]. Available from: https://ais.gov.au/nutrition/supplements/ tiles/group_d

11. Van Breemen RB, Fong HHS, Farnsworth NR. Ensuring the safety of botanical dietary supplements. Am J Clin Nutr. 2008;87(2):509-13.

12. Sprouse AA, Van Breemen RB. Pharmacokinetic interactions between drugs and botanical dietary supplements. Drug Metab Dispos. 2016;44(2):162-71.

13. Alves M, Capela J. Dietary supplements for weight loss with synephrine: risks and toxicity. 2019;16(16):36-46.

14. Gutiérrez-Hellín J, Del Coso J. Effects of p-synephrine and caffeine ingestion on substrate oxidation during exercise. Med Sci Sports Exerc. 2018;50(9):1899-906.

15. Haller CA, Benowitz NL, Jacob P. Hemodynamic effects of ephedra-free weight-loss supplements in humans. Am J Med. 2005;118(9):998-1003.

16. Stohs S. Safety, efficacy, and mechanistic studies regarding citrus aurantium (bitter orange) extract and p-synephrine. Phyther Res. 2017;31(10):1463-74.

17. Shara M, Stohs SJ, Smadi MM. Safety evaluation of p-synephrine following 15 days of oral administration to healthy subjects: A clinical study. Phyther Res 2018 [cited 2020 Jun 21];32(1):125-31. Available from: https://pubmed.ncbi. nlm.nih.gov/29130542/

18. Schmitt GC, Arbo MD, Lorensi AL, Jacques ALB, Nascimento SN, Mariotti KC, et al. Gender differences in biochemical markers and oxidative stress of rats after 28 days oral exposure to a mixture used for weight loss containing p-synephrine, ephedrine, salicin, and caffeine. Brazilian J Pharm Sci. 2016;52(1):59-68. https://doi.org/10.1590/ S1984-82502016000100007

19. Arbo MD, Schmitt GC, Limberger MF, Charão MF, Moro ÂM, Ribeiro GL, et al. Subchronic toxicity of Citrus aurantium L. (Rutaceae) extract and p-synephrine in mice. Regul Toxicol Pharmacol. 2009;54(2):114-7.

20. Schmitt GC, Arbo MD, Lorensi AL, Maciel ÉS, Krahn CL, Mariotti KC, et al. Toxicological effects of a mixture used in weight loss products: P-synephrine associated with ephedrine, salicin, and caffeine. Int J Toxicol. 2012 [cited 2021 Jun 22];31(2):184-91. Available from: http://ijt.sagepub.com

21. Rossato LG, Costa VM, Limberger RP, Bastos ML, Remião F. Synephrine: from trace concentrations to massive consumption in weight-loss. Food Chem Toxicol. 2011;49(1):8-16. https://doi.org/10.1016/j.fct.2010.11.007

22. Chen P, Bryden N. Determination of yohimbine in yohimbe bark and related dietary supplements using UHPLC-UV/ MS: Single-laboratory validation. J AOAC Int. 2015;98(4):896-901.

23. Tam SW, Worcel M, Wyllie M. Yohimbine: A clinical review. Pharmacol Ther. 2001;91(3):215-43.

24. Semwal RB, Semwal DK, Vermaak I, Viljoen A. A comprehensive scientific overview of Garcinia cambogia. Fitoterapia. 2015;102:134-48. https://doi.org/10.1016/j.fitote.2015.02.012

25. Haber S, Awwad O, Phillips A, Park A, Pham T. Garcinia Cambogia: safe for weight loss? Am J Heal Pharm. 2018[cited 2020 Jun 21];75(2):17-22. Available from: http://www.webmd.com/vitamins-and-supplements/garcinia-cambogiaweight-loss\#1 
26. Yu JS, Choi MS, Park JS, Rehman SU, Nakamura K, Yoo HH. Inhibitory effects of Garcinia cambogia Extract on CYP2B6 Enzyme Activity. Planta Med. 2017;83(11):895-900.

27. Sharma A, Akagi E, Njie A, Arsene C, Krishnamoorthy G, Ehrinpreis M. Acute hepatitis due to garcinia cambogia, an herbal weight loss supplement. Case Reports Gastrointest Madicines. 2018;112:S1288.

28. Antidopagem M, Rebelo P. Lista de Substâncias e Métodos Proibidos. 2019;1-13.

29. Krentz AJ, Fujioka K, Hompesch M. Evolution of pharmacological obesity treatments: focus on adverse side-effect profiles. Diabetes Obes Metab. 2016;18(6):558-70.

30. Pickering C, Kiely J. Are the current guidelines on caffeine use in sport optimal for everyone? Inter-individual variation in caffeine ergogenicity, and a move towards personalised sports nutrition. Sport Med. 2018;48(1):7-16.

31. Trexler ET, Smith-Ryan AE. Creatine and caffeine: considerations for concurrent supplementation. Int J Sport Nutr Exerc Metab. 2015;25(6):607-23.

32. Southward K, Rutherfurd-Markwick KJ, Ali A. The effect of acute caffeine ingestion on endurance performance: a systematic review and meta-analysis. Sport Med. 2018;48(8):1913-28. https://doi.org/10.1007/s40279-018-0939-8

33. Temple JL, Bernard C, Lipshultz SE, Czachor JD, Westphal JA, Mestre MA. The safety of ingested caffeine: a comprehensive review. Front Psychiatry. 2017;8(80):1-19.

34. Lopes M, Capela J. Study on the composition of thermogenic food supplements containing caffeine available in Portugal. Assoc Port Nutr. 2017;10(10):24-36.

35. Crisafulli DL, Buddhadev HH, Brilla LR, Chalmers GR, Suprak DN, San Juan JG. Creatine-electrolyte supplementation improves repeated sprint cycling performance: a double blind randomized control study. J Int Soc Sports Nutr. 2018;15(21):1-11.

36. Kreider RB, Kalman DS, Antonio J, Ziegenfuss TN, Wildman R, Collins R, et al. International Society of Sports Nutrition position stand: safety and efficacy of creatine supplementation in exercise, sport, and medicine. J Int Soc Sports Nutr. 2017;14(1):1-18.

37. Antonio J, Candow DG, Forbes SC, Gualano B, Jagim AR, Kreider RB, et al. Common questions and misconceptions about creatine supplementation: what does the scientific evidence really show? J Inter Soc Sports Nutr.; 2021;18:117. https://doi.org/10.1186/s12970-021-00412-w

38. Hall M, Trojian TH. Creatine supplementation. Curr Sports Med Rep. 2013 Jul;12(4):240-4.

39. Jagim AR, Stecker RA, Harty PS, Erickson JL, Kerksick CM. Safety of creatine supplementation in active adolescents and youth: a brief review. Front Nutr. 2018;5(115):1-13.

40. Rust P, Ekmekcioglu C. Impact of salt intake on te pathogenesis and treatment of hypertension. Advs Exp Med Biol Respir. 2016;10:61-84.

41. Slifman N, Obermeyer W, Musser S, Correl W, Joseph S, Love L. Contamination of botanical dietary supplements by digitalis lanata. N Engl J Med. 1998;339(12):806-11.

42. Sharma A, Akagi E, Njie A, Goyal S, Arsene C, Krishnamoorthy G, et al. Acute hepatitis due to garcinia cambogia extract, an herbal weight loss supplement. Case Rep Gastrointest Med. 2018;2018:1-4. 